\title{
Nanotubes based on monolayer blue phosphorus
}

\author{
E. Montes and U. Schwingenschlögl* \\ King Abdullah University of Science and Technology (KAUST), Physical Science and Engineering Division (PSE), \\ Thuwal 23955-6900, Saudi Arabia
}

(Received 8 March 2016; revised manuscript received 18 June 2016; published 8 July 2016)

\begin{abstract}
We demonstrate structural stability of monolayer zigzag and armchair blue phosphorus nanotubes by means of molecular dynamics simulations. The vibrational spectrum and electronic band structure are determined and analyzed as functions of the tube diameter and axial strain. The nanotubes are found to be semiconductors with a sensitive indirect band gap that allows flexible tuning.
\end{abstract}

DOI: 10.1103/PhysRevB.94.035412

\section{INTRODUCTION}

Layered structures based on $\mathrm{P}$ very recently are attracting considerable attention [1,2], initiated by research on black phosphorus, the most stable $\mathrm{P}$ allotrope [3] and the only elemental two-dimensional material besides graphene that can be mechanically exfoliated [4]. Black phosphorus consists of layers of hexagonally arranged $\mathrm{P}$ atoms with two different P-P bond lengths that are held together by weak van der Waals forces, thus resembling graphite, but with a puckered structure. Phosphorene, a monolayer of black phosphorus, has a high carrier mobility of up to $\sim 1000 \mathrm{~cm}^{2} / \mathrm{Vs}$ [5] and a direct band gap, which is tunable by means of strain and shrinks from $1.5 \mathrm{eV}$ for a monolayer to $0.6 \mathrm{eV}$ for a five-layer slab [6]. The size of the band gap lies between those of graphene (zero) and the transition metal dichalcogenides (1.5 to $2.5 \mathrm{eV})$ [7]. A distinct directional anisotropy between the zigzag and armchair directions ( $\sim 2$ for holes and $\sim 4$ for electrons) has been reported in Ref. [8]. Phosphorene has been put forward as a candidate for high performance thin film electronics and already has been used for fabricating reliable field effect transistors [9].

It has been demonstrated theoretically that specific dislocations of the atoms in the phosphorene lattice can generate additional layered phases $(\beta-\mathrm{P}, \gamma-\mathrm{P}$, and $\delta$-P) $[10,11]$. Of particular interest is the blue phosphorus allotrope $(\beta-\mathrm{P})$, which is nearly as stable as black phosphorus and has a honeycomb lattice with buckled structure (not puckered), showing a largely isotropic thermal conductivity [12]. Dirac cones have been predicted for hydrogenated and fluorinated $\beta$-P under tensile strain [13]. Although $\beta$-P has not yet been synthesized, it has been proposed to be used as anode for high capacity Li ion batteries [14]. While intense research has aimed at understanding the electronic and transport properties of two-dimensional layered structures based on $\mathrm{P}$ $[15,16]$, one-dimensional structures rarely have been explored. Nanotubes based on phosphorene have been introduced in Ref. [17] and the electronic properties of $\beta$-P nanotubes have been discussed in Refs. $[18,19]$. In the latter works round tubes have been generated by rolling up pristine $\beta$-P and faceted nanotubes by creating defect lines. While it turns out that faceted nanotubes are energetically favorable for small diameters, round nanotubes do not spontaneously transform

\footnotetext{
*udo.schwingenschlogl@kaust.edu.sa
}

into faceted geometries at elevated temperature. In addition, it is expected that round nanotubes are energetically favorable for large diameters. Since the stability so far has been investigated only by means of phonon calculations, we study in the present work monolayer zigzag and armchair $\beta$-P nanotubes by means of molecular dynamics simulations. In addition, the phonon spectrum and electronic band structure are analyzed as functions of the tube diameter and axial strain.

\section{METHODOLOGY}

Using density functional theory as implemented in the SIESTA code [20,21], we optimize the structure and calculate the phononic and electronic dispersion relations of $\beta-\mathrm{P}$ nanotubes. Three-dimensional periodic boundary conditions are applied, which give rise to a tube in the $z$-direction but require the addition of a sufficiently thick vacuum region in the $x y$-plane to avoid artificial interaction with image tubes. We employ double zeta plus polarization basis sets, describe the core electrons by norm-conserving TroullierMartins pseudopotentials [22], and calculate the exchange correlation functional within the local density approximation (Ceperley-Alder parametrization [23]). The reciprocal space is sampled on a Monkhorst-Pack $1 \times 1 \times 10 \mathrm{k}$-mesh and the real space mesh is determined by an energy cutoff of 200 Ry. For calculating the vibrational spectrum we use a supercell consisting of 5 unit cells of the nanotube along the axial direction. The stability of the nanotubes is verified by calculations of the vibrational spectra as well as by finite temperature molecular dynamics simulations, using the Nosé algorithm (since a structure with stable phonon spectrum still can become unstable by thermal fluctuations at elevated temperature). The same procedure has been used previously to determine the stability of several other structures [10,24]. A supercell consisting of 5 unit cells is found to be sufficient to converge the total energy.

\section{RESULTS AND DISCUSSION}

Nanotubes are built by rolling up $\beta$-P such that either the zigzag or armchair direction corresponds to the tube axis. For different diameters $D$, we consider zigzag $(n, 0)$ and armchair $(n, n)$ nanotubes, specifically $(5,0)$ with $D=7.6 \AA$, $(6,0)$ with $D=7.9 \AA,(7,0)$ with $D=9.4 \AA,(8,0)$ with $D=9.9 \AA,(9,0)$ with $D=11.2 \AA$, and $(10,0)$ with $D=11.9 \AA$ as well as $(5,5)$ with $D=10.5 \AA$, $(6,6)$ with $D=12.2 \AA$, $(7,7)$ with $D=$ 


\section{Blue Phosphorus ( $\beta-\mathrm{P})$}

Top view

\section{Zigzag nanotube}

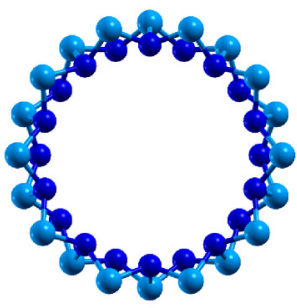

Cross-section

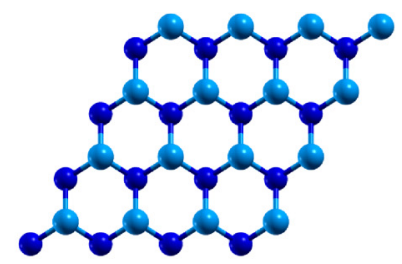

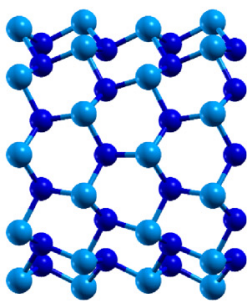

Side view

\section{Armchair nanotube}

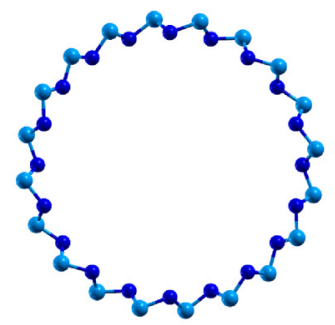

Cross-section

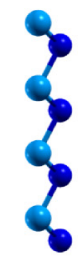

Side view

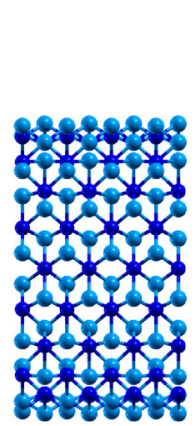

Side view

FIG. 1. Structures of $\beta$-P as well as zigzag $(10,0)$ and armchair $(10,10) \beta$-P nanotubes. The two atomic layers are distinguished by color.

$14.0 \AA$, $(8,8)$ with $D=15.7 \AA$, $(9,9)$ with $D=17.6 \AA$, and $(10,10)$ with $D=19.2 \AA$. All structures are fully relaxed using the conjugated gradient method [25], until the residual forces on all atoms have declined below $0.02 \mathrm{eV} / \AA$. The reference structure of $\beta$-P is presented in Fig. 1 (top) in top and side views. The buckling of the honeycomb lattice is illustrated using different colors for the atoms in the two sublattices. Cross-section and side views are given in Figs. 1 (middle) and 1 (bottom) for the zigzag $(10,0)$ and armchair $(10,10)$ nanotubes, respectively. Due to the buckling, there are two classes of atoms with small and large distances to the tube axis. The curvature energy $E_{c}$ (formation energy) is defined as the energy difference per atom between a nanotube and $\beta$-P. Our results in Fig. 2 show that $E_{c}$ is positive and increases with the curvature. In accordance with the classical theory of elasticity [26], we find in good approximation $E_{c} \propto 1 / D^{2}$.

Our molecular dynamics simulations cover time periods of at least 2 ps with time steps of $1 \mathrm{fs}$. Each system is first equilibrated for 3000 steps at $0 \mathrm{~K}$ before the temperature is increased in steps of $150 \mathrm{~K}$ or $300 \mathrm{~K}$. In each step the system

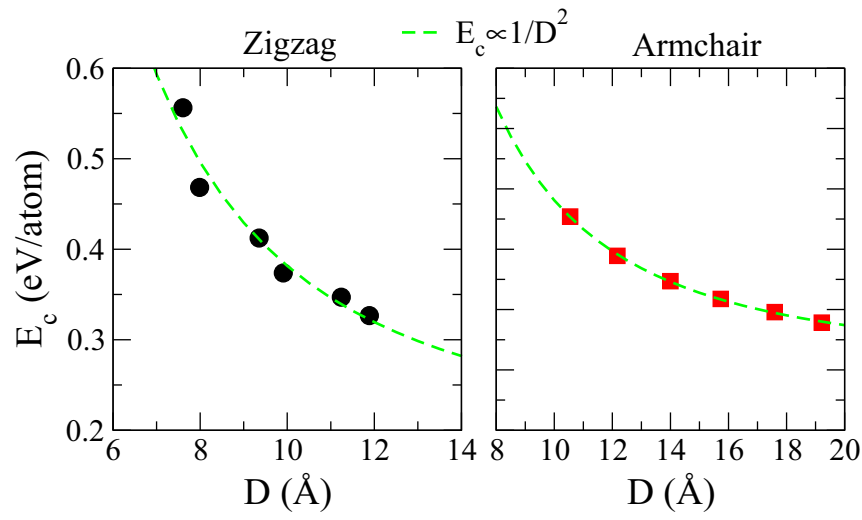

FIG. 2. Curvature energy as a function of the tube diameter. Fitting curves are given by green dashed lines.

Zigzag $(8,0)$
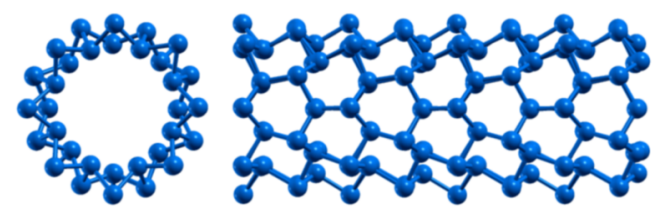

$\mathrm{T}=300 \mathrm{~K}$

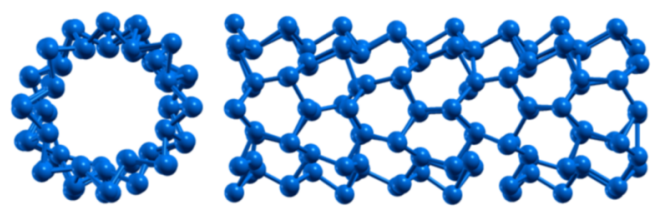

$\mathrm{T}=900 \mathrm{~K}$

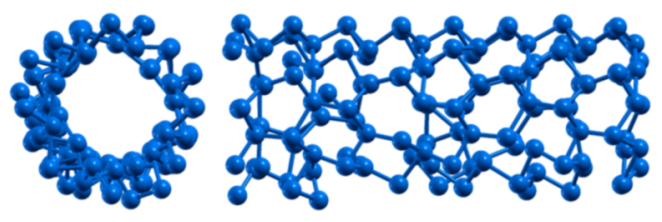

$\mathrm{T}=1500 \mathrm{~K}$
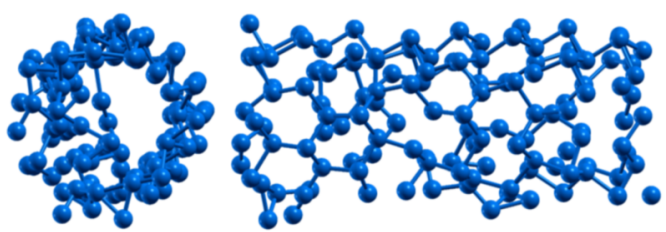

$\mathrm{T}=2100 \mathrm{~K}$
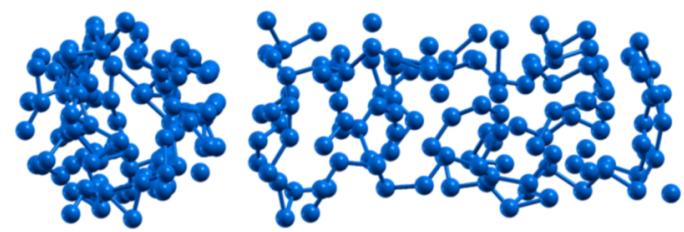

$\mathrm{T}=2700 \mathrm{~K}$

FIG. 3. Snapshots from the molecular dynamics simulation of the zigzag $(8,0) \beta$-P nanotube at increasing temperatures. 
is equilibrated for at least 2000 steps. In Fig. 3 we present as an example snapshots of the zigzag $(8,0) \beta$-P nanotube after equilibration at increasing temperatures. From $300 \mathrm{~K}$ to $1500 \mathrm{~K}$ the nanotube shows growing structural fluctuations both in the cross-section and side views. At $2100 \mathrm{~K}$ it starts to deform and at $2700 \mathrm{~K}$ the structural integrity is largely lost. In order to estimate the melting temperature we follow the approach of Refs. [27,28]. The converged potential energy of the molecular dynamics simulations is plotted as a function of the temperature in Fig. 4 (left) for different nanotubes. Melting is reflected by deviation from a linear dependence. The estimated melting temperatures summarized in Fig. 4 (right) show an increase with the tube diameter, as expected from the decreasing curvature. Our results indicate that $\beta$-P nanotubes are not only stable at $300 \mathrm{~K}$ but also above the melting temperature of bulk phosphorus (863 K [29]). The inset of Fig. 4 demonstrates that the employed methodology results in a melting temperature of $\sim 825 \mathrm{~K}$ for bulk phosphorus, in good agreement with the experiment.

The phonon dispersion is presented in Fig. 5 (left) from the $\Gamma$ point $\left(k_{z}=0\right)$ to the $\mathrm{Z}$ point $\left(k_{z}=\pi / a\right)$. The nanotubes are stable in the sense that all frequencies are positive, the acoustic and optical modes being well separated in both the zigzag and armchair cases. This separation depends on $D$, being hardly noticeable for small zigzag nanotubes and comparable to that of $\beta$-P, see Fig. 6, for large armchair nanotubes. The hardness of the longitudinal optical modes observed in $\beta$-P [10] is even slightly increased in the nanotubes.

In any tubular nanostructure the acoustic modes are determined by breathing (radial translations of the atoms) and twiston (torsion of the tube) distortions [30,31]. The
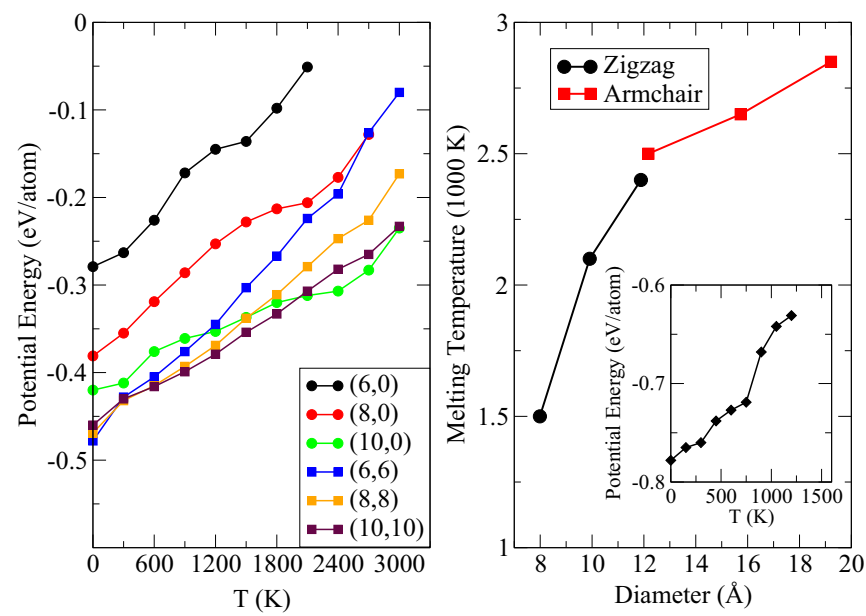

FIG. 4. Left: Potential energy per atom as function of the temperature for zigzag $(6,0),(8,0),(10,0)$ and armchair $(6,6),(8,8)$, $(10,10) \beta$-P nanotubes. Right: Melting temperature as function of the tube diameter. The inset shows the temperature dependence of the potential energy of bulk phosphorus.

breathing mode is identified in Fig. 5 (left) at low frequency (red color), as expected from its radial nature. The frequency at the $\Gamma$ point turns out to decrease as $D$ increases. The twiston mode is required to have zero frequency at the $\Gamma$ point [32] and is highlighted in Fig. 5 (left) by green color. Its dispersion is almost linear close to the $\Gamma$ point. The other two acoustic modes correspond to translations of the nanotube perpendicular to the tube axis and do not appear for the

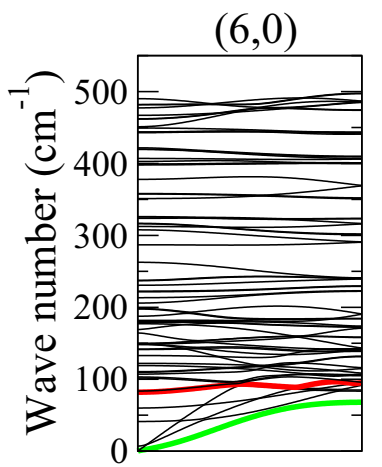

$(6,6)$

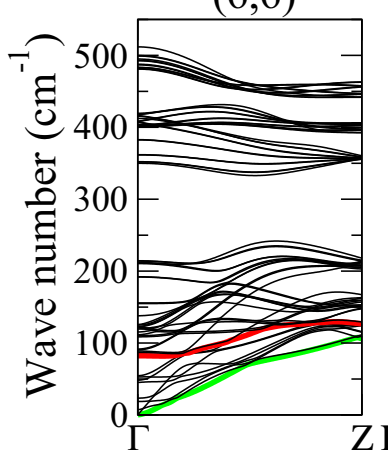

$(8,0)$

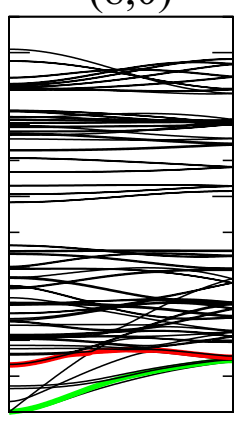

$(8,8)$

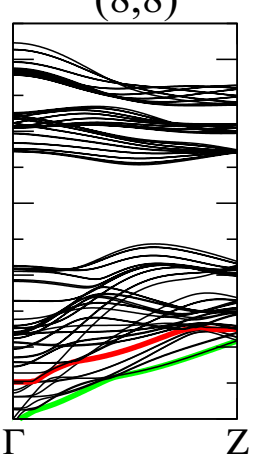

$(10,0)$

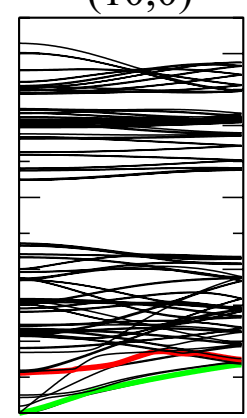

$(10,10)$

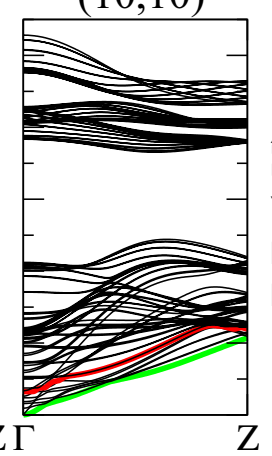

$(6,0)$

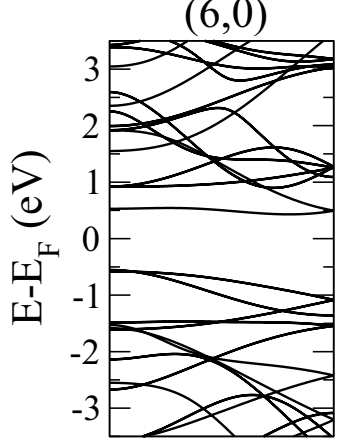

$(6,6)$

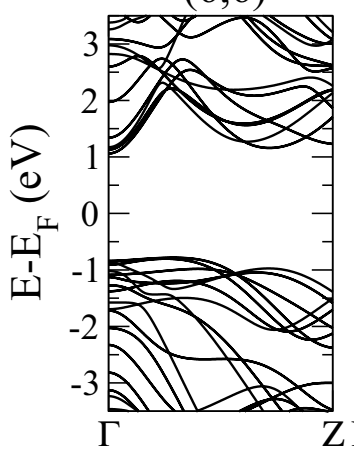

$(8,0)$

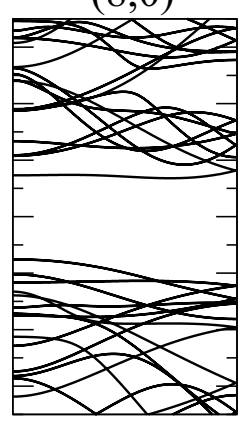

$(8,8)$

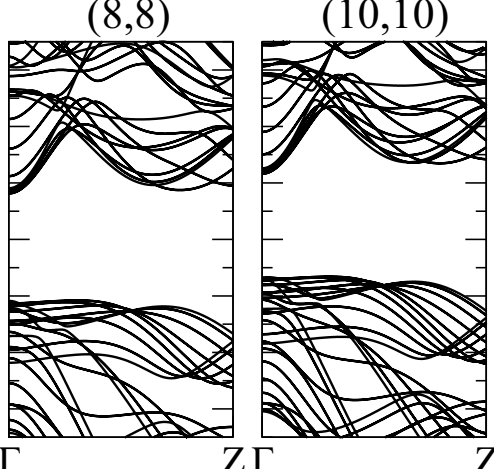

$(10,0)$

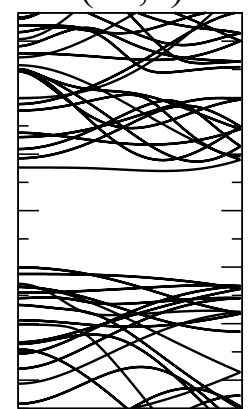

$(10,10)$

FIG. 5. (Left) Vibrational spectrum and (right) electronic band structure of (top) zigzag and (bottom) armchair $\beta$-P nanotubes. Color is used to mark the breathing (red) and twiston (green) modes. 

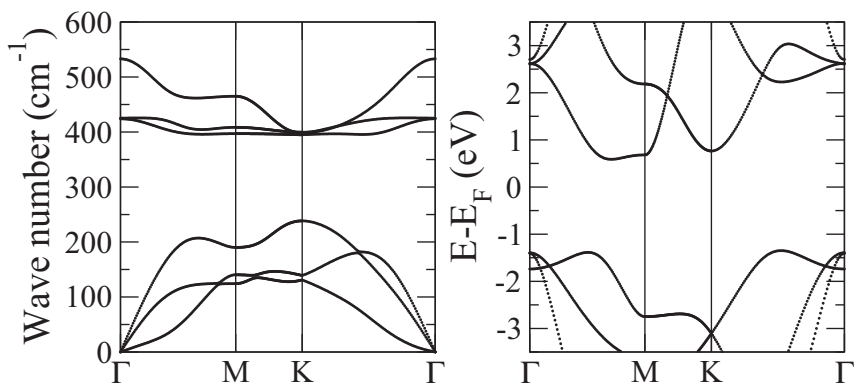

FIG. 6. (Left) Vibrational spectrum and (right) electronic band structure of $\beta$-P in the hexagonal Brillouin zone.

monolayer. While the low frequency modes describe the radial and torsional properties of the nanotube, the intermediate and high frequency modes are inherited from $\beta$-P and accordingly depend on the chirality. We observe that particularly the high frequency modes become stiffer for increasing $D$ due to the decreasing curvature.

The band structure of $\beta$-P in Fig. 6 (right) shows an indirect band gap of about $2 \mathrm{eV}$, with the valence band maximum located along the $\Gamma-\mathrm{K}$ direction (armchair direction) and the conduction band minimum along the $\Gamma-\mathrm{M}$ direction (zigzag direction). The band structures of the nanotubes given in Fig. 5 (right) are also characterized by indirect band gaps ( $\Gamma-\mathrm{Z}$ correspond to the tube axis). For the zigzag nanotubes the valence band maximum appears at the $\Gamma$ point and the conduction band minimum at about $65 \%$ of $\Gamma-\mathrm{Z}$, while for the armchair nanotubes the valence band maximum is located at about $25 \%$ of $\Gamma-\mathrm{Z}$ and the conduction band minimum at the $\Gamma$ point. Since the $\Gamma-\mathrm{M}$ and $\Gamma-\mathrm{K}$ directions of $\beta$-P, see Fig. 6 (right), correspond to the $\Gamma-\mathrm{Z}$ directions of the zigzag and armchair nanotubes, respectively, it turns out that the valence band edge of $\beta$-P qualitatively resembles that of the armchair nanotube and the conduction band edge of $\beta$-P that of the zigzag nanotube.

The dependence of the band gap size on $D$ is shown in Fig. 7 (left), in comparison to $\beta$-P. For the nanotube with the smallest
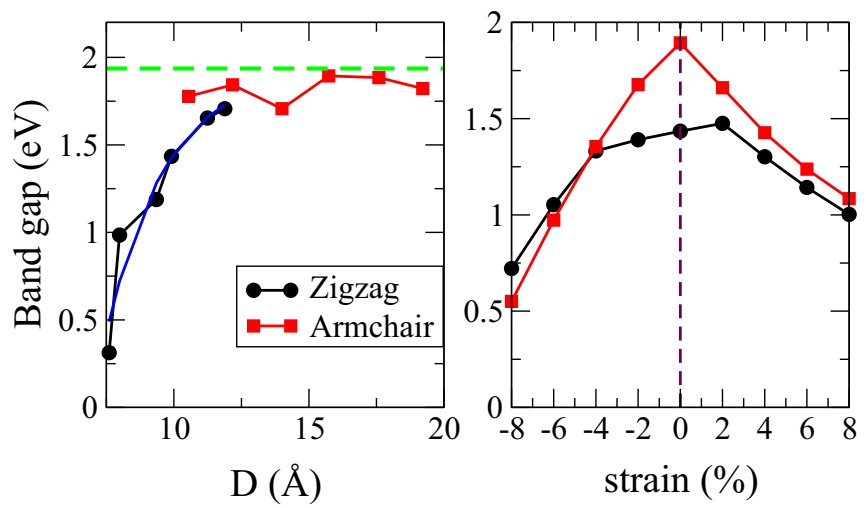

FIG. 7. (Left) Dependence of the band gap size on the nanotube diameter. The green dashed line refers to the band gap of $\beta$-P and the blue line is obtained by exponential fitting. (Right) Dependence of the band gap size on axial strain for the zigzag $(8,0)$ and armchair $(8,8)$ nanotubes.
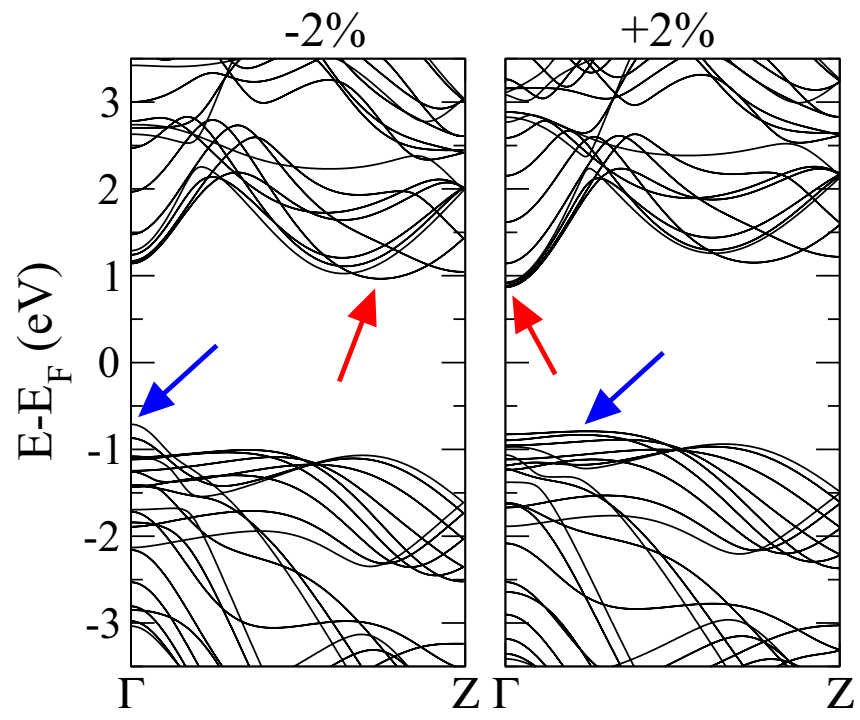

FIG. 8. Electronic band structure of the armchair $(8,8) \beta$-P nanotube under $-2 \%$ (compressive) and $+2 \%$ (tensile) axial strain. The valence and conduction band edges are marked by blue and red arrows, respectively.

$D$ the separation between opposite $\mathrm{P}$ atoms $(5.3 \AA)$ is similar to the interlayer distance in black phosphorus $(5.5 \AA)$ and the band gap $(0.31 \mathrm{eV})$ also resembles that of black phosphorus $(\sim 0.3 \mathrm{eV})$ [1]. The larger the nanotube, the smaller is the curvature and the larger is the distance between opposite $\mathrm{P}$ atoms. We find that the band gap grows strongly for increasing $D$ in the case of the zigzag nanotubes, while remaining almost constant for the armchair nanotubes. However, in both cases it approaches the band gap of $\beta-\mathrm{P}(1.93 \mathrm{eV})$ for large $D$. For the zigzag nanotube the dependence of the band gap can be fitted as $E_{\text {gap }}=1.93 \mathrm{eV}-a e^{-b D}$, with parameters $a$ and $b$. It is important to notice that in layered phosphorus systems the band gap decreases with the thickness, exponentially when they are based on phosphorene [8] and linearly when they are based on $\beta$-P [10]. For phosphorene nanotubes the band gap also approaches exponentially the value of the corresponding monolayer for growing $D$ [17].

Another similarity is the sensitive dependence of the band gap on axial strain, which is useful in electronic applications [33]. For strain between $-8 \%$ (compression) and $+8 \%$ (tensile) this dependence is shown in Fig. 7 (right) for the zigzag $(8,0)$ and armchair $(8,8) \beta$-P nanotubes (structure optimized for each value of the strain). Interestingly, the maximal band gap is obtained in the pristine cases ( $0 \%$ strain). A similarly strong dependence (with the maximum close to $0 \%$ strain) has been reported for $\beta$-P [10], whereas for phosphorene nanotubes the band gap grows continuously from $-4 \%$ to $4 \%$ strain [17].

In order to analyze the abrupt change from growing and shrinking band gap in the case of the armchair $(8,8)$ nanotube, see Fig. 7 (right), we show in Fig. 8 electronic band structures under $-2 \%$ and $+2 \%$ axial strain. We find transitions for both the valence and conduction band edges, see the blue and red arrows, which explain the observed behavior. 


\section{CONCLUSION}

In conclusion, we have demonstrated structural stability of nanotubes based on $\beta$-P for a wide range of diameters and both chiralities (zigzag or armchair direction along the tube axis). Because of the weak interlayer interaction in bulk phosphorus, it is reasonable to conjecture that such nanotubes indeed can be grown [3]. Our molecular dynamic simulations cover time periods of at least 1 ps. As compared to room temperature, some structural modifications are evident at $1200 \mathrm{~K}$ but no structural transition occurs, only the onset of a melting process is observed. Therefore $\beta-\mathrm{P}$ nanotubes are not only stable at $300 \mathrm{~K}$ but also above the melting temperature of bulk phosphorus $(\geqslant 863 \mathrm{~K})$. All nanotubes under consideration are found to be indirect band gap semiconductors, with the shape of the valence/conduction band of the armchair/zigzag nanotube qualitatively reproducing that of $\beta$-P. The size of the band gap is found to be very sensitive to the tube diameter, exponentially approaching the value of $\beta$-P, for the zigzag nanotubes and almost constant, slightly smaller than in $\beta-\mathrm{P}$, for the armchair nanotubes. The maximal band gap in both cases is found when no strain is applied, decreasing quickly under compression as well as tension. By this flexibility of the band structure, nanotubes formed from $\beta$-P have great potential as a one-dimensional platform for electronic devices.

\section{ACKNOWLEDGMENT}

The research reported in this publication was supported by funding from King Abdullah University of Science and Technology (KAUST).
[1] X. Ling, H. Wang, S. Huang, F. Xia, and M. S. Dresselhaus, Proc. Natl. Acad. Sci. USA 112, 4523 (2015).

[2] H. Liu, Y. Du, Y. Deng, and P. D. Ye, Chem. Soc. Rev. 44, 2732 (2015).

[3] H. Liu, A. T. Neal, Z. Zhu, Z. Luo, X. Xu, D. Tománek, and P. D. Ye, ACS Nano 8, 4033 (2014).

[4] W. Lu, H. Nan, J. Hong, Y. Chen, C. Zhu, Z. Liang, X. Ma, Z. Ni, C. Jin, and Z. Zhang, Nano Res. 7, 853 (2014).

[5] S. P. Koening, R. A. Doganov, H. Schmidt, A. H. Castro Neto and B. Özyilmaz, Appl. Phys. Lett. 104, 103106 (2014).

[6] R. Fei and L. Yang, Nano Lett. 14, 2884 (2014).

[7] Y. Du, C. Ouyang, S. Shi and M. Lei. J. Appl. Phys. 107, 093718 (2010).

[8] J. Qiao, X. Kong, Z.-X. Hu, F. Yang, and W. Ji, Nat. Commun. 5, 4475 (2014).

[9] L. Li, Y. Yu, G. J. Ye, Q. Ge, X. Ou, H. Wu, D. Feng, X. H. Chen, and Y. Zhang, Nat. Nanotechnol. 9, 372 (2014).

[10] Z. Zhu and D. Tománek, Phys. Rev. Lett. 112, 176802 (2014).

[11] J. Guan, Z. Zhu, and D. Tománek, Phys. Rev. Lett. 113, 046804 (2014).

[12] A. Jain and A. J. H. McGaughey, Sci. Rep. 5, 8501 (2015).

[13] Y. Li and X. Chen, 2D Materials 1, 031002 (2014).

[14] Q.-F. Li, C.-G. Duan, X. G. Wan, and J.-L. Kuo, J. Phys. Chem. C 119, 8662 (2015).

[15] A. S. Rodin, A. Carvalho and A. H. Castro Neto, Phys. Rev. Lett. 112, 176801 (2014).

[16] F. Xia, H. Wang, and Y. Jia, Nat. Commun. 5, 4458 (2014).

[17] H. Guo, N. Lu, J. Dai, X. Wu, and X. C. Zeng, J. Phys. Chem. C 118, 14051 (2014).

[18] J. Xiao, M. Long, C.-S. Deng, J. He, L.-H. Cui, and H. Xu, J. Phys. Chem. C 120, 4638 (2016).
[19] Y. Aierken, O. Leenaerts, and F. M. Peeters, Phys. Rev. B 92, 104104 (2015).

[20] D. Sánchez-Portal, P. Ordejón, E. Artacho, and J. M. Soler, Int. J. Quantum Chem. 65, 453 (1997).

[21] J. M. Soler, E. Artacho, J. D. Gale, A. García, J. Junquera, P. Ordejón, and D. Sánchez-Portal, J. Phys.: Condens. Matter 14, 2745 (2002).

[22] N. Troullier and J. L. Martins, Phys. Rev. B 43, 1993 (1991).

[23] D. M. Ceperley and B. J. Alder, Phys. Rev. Lett. 45, 566 (1980).

[24] S. Cahangirov, M. Topsakal, E. Aktürk, H. Şahin and S. Ciraci, Phys. Rev. Lett. 102, 236804 (2009).

[25] M. R. Hestenes and E. Stiefel, J. Res. Natl. Bur. Stand. 49, 409 (1952).

[26] O. Gülseren, T. Yildirim, and S. Ciraci, Phys. Rev. B 65, 153405 (2002).

[27] S. B. Fagan, R. Mota, R. J. Baierle, G. Paiva, A. J. R. da Silva and A. Fazzio, J. Mol. Struct. 539, 101 (2001).

[28] S. J. Wang, C. L. Zhang and Z. G. Wang, Chin. Phys. Lett. 27, 106101 (2010).

[29] C. Kittel, Introduction to Solid State Physics, 8th ed. (John Wiley \& Sons Inc., Hoboken, NJ, 2005).

[30] D. Sánchez-Portal, E. Artacho, J. M. Soler, A. Rubio, and P. Ordejon, Phys. Rev. B 59, 12678 (1999).

[31] R. A. Jishi, L. Venkataraman, M. S. Dresselhaus, and G. Dresselhaus, Chem. Phys. Lett. 209, 77 (1993).

[32] C. L. Kane, E. J. Mele, R. S. Lee, J. E. Fischer, P. Petit, H. Dai, A. Thess, R. E. Smalley, A. R. M. Verschueren, S. J. Tans, and C. Dekker, Europhys. Lett. 41, 683 (1998).

[33] Y. Li, S. Yang, and J. Li, J. Phys. Chem. C 118, 23970 (2014). 